\title{
Informes
}

\section{La agenda futura de investigación sobre trabajo no remunerado y familia}

\author{
Relatoría de Isalia Nava Bolaños*
}

En el marco de la celebración de los 50 años del Centro de Estudios Demográficos, Urbanos y Ambientales (CEDUA) y como parte del Seminario Permanente de Diálogos y Reflexiones sobre Población, Ciudad y Medio Ambiente, el 28 de febrero de 2014 se presentó la sesión "La agenda futura de investigación sobre trabajo no remunerado y familia" en el auditorio Alfonso Reyes de El Colegio de México. El interés primordial de esta sesión, que fue organizada por Brígida García y Edith Pacheco, investigadoras adscritas a esta institución, fue escuchar los planteamientos de María de la Paz López Barajas (asesora regional de la Entidad de la Organización de las Naciones Unidas para la Igualdad de Género y el Empoderamiento de la Mujer-ONU Mujeres-), de María Ángeles Durán (profesora de Sociología e investigadora en el Departamento de Economía del Centro de Ciencias Humanas y Sociales en el Consejo Superior de Investigaciones Científicas de España-CSIC-) y de Paulo Saad (jefe del Área de Población y Desarrollo del Centro Latinoamericano y Caribeño de Demografía-Celade-, División de Población de la Comisión Económica para América Latina y el Caribe -CEPAL-) en relación con el trabajo no remunerado, la familia y la economía global. Asimismo se pretendió generar un espacio en donde se reflexionara sobre los acercamientos teóricos y metodológicos, los resultados empíricos y los temas aún pendientes que marcarán las líneas futuras de investigación. En esta relatoría se ofrece una síntesis de las presentaciones de los autores y se resumen las discusiones y reflexiones que éstas generaron entre el público asistente.

\section{The Future Research Agenda on Unpaid Work and Family}

As part of the celebration of the 50th anniversary of the Center for Demographic, Urban and Environmental Studies (CEDUA) and the Permanent Seminar of Dialogues and Reflections on Population, City and Environment, on February 28, 2014, the session on "The Future Research Agenda for Unpaid Work and Family" was held in the Alfonso Reyes auditorium at El Colegio de México. The primary focus of this meeting, organized by Brigida García and Edith Pacheco, researchers affiliated to this institution, was to hear the proposals of Maria de la Paz López Barajas (regional advisor to the

* Investigadora en el Instituto de Investigaciones Económicas, UNAM. Dirección postal: Circuito Mario de la Cueva s/n, Ciudad Universitaria, C.P. 04510, México, D.F., México. Correo electrónico:<isalia.nava@iiec.unam.mx>.

$\overline{\text { ESTUDIOS DEMOGRÁFICOS Y URBANOS, VOL. 29, NÚM. } 3 \text { (87), 2014, 621-635 }}$ 
United Nations Entity for Gender Equality and the Empowerment of Women -UN Women-), Maria Angeles Durán (sociology professor and researcher at the Department of Economics of the Center for Human and Social Sciences at the Higher Council for Scientific Research of Spain-CSIC-) and Paulo Saad (Head of the Department of Population and Development of the Latin American and Caribbean Center of Demography -Celade-, at the Population Division of the Economic Commission for Latin America and the Caribbean-CEPAL-) in relation to unpaid work, family and the global economy. The aim was also to create a space for reflection on theoretical and methodological approaches, empirical results and pending issues that will shape future research. This report provides a summary of the authors' presentations and summarizes the discussions and reflections they elicited among the audience.

\section{Presentación}

Silvia Giorguli, directora del CEDUA, dio la bienvenida a los asistentes; se refirió especialmente a Paulo Saad y a María Ángeles Durán, egresados de la Maestría en Demografía, pues reconoció que su presencia fortalece los vínculos con otras comunidades que están dialogando sobre estos mismos temas. Asimismo agradeció a Brígida García y a Edith Pacheco la iniciativa de organizar dicha sesión y enfatizó la importancia de abordar esta temática.

Destacó que se trata de una línea de investigación que ha estado presente a lo largo de los 50 años del Centro y no solamente se ha abocado a estudiar los mercados de trabajo desde una perspectiva demográfica, sino también su vinculación con el tema de la familia. Un primer antecedente se ubica en 1979 con la publicación del texto Migración, familia y fuerza de trabajo en la Ciudad de México, de Brígida García, Humberto Muñoz y Orlandina de Oliveira. A lo largo de los años esta línea se ha mantenido como un tema de discusión, investigación y publicación del Centro, donde los trabajos de Brígida García y sus publicaciones en colaboración con Orlandina de Oliveira son referentes clásicos. Silvia Giorguli expuso que además de la investigación, en el CEDUA se promueven la formación y la participación de los egresados en esa línea, y así hay actualmente una escuela muy hecha en cuanto a la formación y los estudios sobre investigación del mercado de trabajo y sobre la perspectiva demográfica en México. Mercedes Pedrero y Edith Pacheco son un claro ejemplo de dicha participación.

La doctora Giorguli mencionó que a pesar de que este tema se ha venido trabajando desde los setenta no se ha agotado, pues han surgido nuevas perspectivas y se han puesto en práctica nuevas metodologías. 


\section{INFORMES}

Celebró la conclusión del proyecto entre ONU Mujeres y El Colegio de México, que culminará con la publicación del libro Uso del tiempo y trabajo no remunerado en México, coordinado por Brígida García y Edith Pacheco. De acuerdo con la directora del CEDUA, este tipo actividades permiten seguir incursionando y discutiendo sobre nuevas aproximaciones sobre el tema de trabajo y familia dentro de un ambiente de diálogo entre generaciones en el que se han incorporado estudiantes e investigadores.

También, y como parte de la presentación, Brígida García dio la bienvenida a los asistentes, resaltó la relevancia de la sesión como un paso más para visibilizar la importancia del trabajo no remunerado en la economía global y destacó la riqueza de contar con la experiencia de profesionales procedentes de distintas partes del mundo y de diversas instituciones.

\section{Intervención de María de la Paz López Barajas}

Landy Sánchez, profesora investigadora del CEDUA, leyó un texto de María de la Paz López Barajas, ${ }^{1}$ quien por motivos laborales no pudo estar presente. El mensaje de María de la Paz, en nombre de ONU Mujeres, enfatizó la importancia de visibilizar las desigualdades de género, la discriminación y la exclusión que viven las mujeres, así como la de mostrar los obstáculos que enfrentan y que les impiden ampliar sus capacidades, hacer uso de sus libertades y ejercer plenamente sus derechos. Es en este contexto que ONU Mujeres tiene como objetivos primordiales generar y analizar la información sobre uso del tiempo y sobre trabajo remunerado y no remunerado.

En su texto, Paz López destacó la importancia de las conferencias e instrumentos internacionales y resaltó las recomendaciones que de allí se derivan: 1) En la Cuarta Conferencia Mundial sobre la Mujer

\footnotetext{
${ }^{1}$ Es asesora regional de ONU Mujeres. En el ámbito académico ha publicado trabajos sobre temas de familias. En su trayectoria profesional ha sido consultora de diversos organismos internacionales para temas de género, como el Banco Interamericano de Desarrollo (BID), el Programa de las Naciones Unidas para el Desarrollo (PNUD), la CEPAL y el Fondo de las Naciones Unidas para la Infancia (UNICEF), entre otros. Asimismo se ha desempeñado como funcionaria de la administración pública federal, donde ha sido responsable de diversos proyectos, muchos de ellos orientados al avance en la igualdad de género. También participa en organizaciones de la sociedad civil que abogan por los derechos de las mujeres. Es egresada de la Maestría en Demografía de El Colegio de México.
} 
en Beijing en 1995, en su plataforma de acción se instó a los estados parte a desarrollar un conocimiento integral de todas las formas de trabajo y empleo a través del mejoramiento de la recolección de datos y de los métodos de medición; a mejorar la recopilación de datos acerca de la contribución de las mujeres y los hombres a la economía, incluyendo su participación en el sector informal; y a desarrollar una clasificación internacional de actividades para incluirla en las estadísticas sobre el uso del tiempo; así como generar estudios periódicos sobre uso del tiempo y trabajo remunerado y no remunerado. 2) En la recomendación general número 17 de la Convención sobre la Eliminación de Todas las Formas de Discriminación Contra la Mujer se recomendó a los estados parte a alentar y apoyar las investigaciones y los estudios experimentales destinados a medir y valorar el trabajo doméstico no remunerado de las mujeres, así como a adoptar medidas encaminadas a cuantificar su trabajo doméstico no remunerado e incluirlo en el producto nacional bruto (PNB). 3) En las conclusiones del $53^{\circ}$ periodo de sesiones de la Comisión Jurídica y Social de la Mujer en 2009 se instó a los estados parte a reconocer el valor del trabajo no remunerado que se realiza dentro de los hogares y en la sociedad en general, y a adoptar las medidas necesarias para que se le incluya en las políticas, estrategias, planes, presupuestos y sectores. Se trata de marcos internacionales que adquieren relevancia en la medida en que incentivan a los países a generar información y a dar un paso adelante con el objetivo de cumplir con los compromisos adquiridos.

La asesora regional de ONU Mujeres enfatizó la importancia central del trabajo no remunerado de ellas. En el Fondo de Desarrollo de las Naciones Unidas para la Mujer (UNIFEM), con Guadalupe Espinoza al frente, la institución mostró particular interés en el tema e inició un importante proceso que en su momento se denominó "trastocar la producción estadística desde la perspectiva de género". A esta tarea se sumaron Vania Salles, Brígida García, Orlandina de Oliveira, Fernando Cortés y Rosa María Ruvalcaba. María de la Paz mencionó que los encuentros de aquel grupo fueron dedicados, entre otras actividades, a discutir cómo captar información del trabajo no remunerado de las mujeres en un módulo del uso del tiempo expresado en apenas una batería de preguntas. El objetivo era convencer al titular del Instituto Nacional de Estadística y Geografía (INEGI) de la importancia de recopilar esta información en el marco de la Encuesta Nacional de Ingresos y Gastos de los Hogares (ENIGH) de 1996. 


\section{INFORMES}

María de la Paz resaltó el papel pionero de México con el trabajo del INEGI en la generación de estadísticas. En 1996 se realizó la Encuesta Nacional sobre Trabajo, Aportaciones y Uso del Tiempo (ENTAUT), en 1998 y 2002 la Encuesta Nacional sobre Uso del Tiempo (ENUT) y en 2009 la Encuesta Nacional de Uso del Tiempo (ENUT). A diferencia de las anteriores, que se levantaron como módulos de la ENIGH, esta última se realizó de manera independiente. Indicó que actualmente el país está cumpliendo con el compromiso internacional de recopilar de manera regular esta información y la está llevando al terreno de la contabilidad nacional con la elaboración de la Cuenta Satélite del Trabajo no Remunerado de los Hogares en México (CSTNRHM). Los cálculos de esta cuenta revelan que el trabajo no remunerado que realizaron los hogares en 2009 representó $22.6 \%$ del producto interno bruto (PIB), proporción superior a la que entonces representó la industria manufacturera y el sector comercio, 16 y $24 \%$ respectivamente. Además, las mujeres realizan $76.6 \%$ del conjunto del valor de este trabajo.

Asimismo María de la Paz celebró los avances en la región, pues actualmente 19 países de América Latina y el Caribe han incorporado módulos de uso del tiempo, preguntas en encuestas locales, o han levantado encuestas específicas sobre uso del tiempo y trabajo no remunerado. Aquí destacó que desde 2006 se ha contado con el acompañamiento de la División de Asuntos de Género de la CEPAL y con la conformación de un grupo de trabajo de estadísticas de género en el seno de la Conferencia de Estadísticas de las Américas, presidido desde entonces por el INEGI. Como producto de estos esfuerzos actualmente se cuenta con herramientas técnico-metodológicas como la Clasificación de Actividades de Uso de Tiempo para América Latina (CAUTAL).

Paz López enfatizó la labor de la academia para incidir en la incorporación del género, en la generación y análisis de la información estadística, y reconoció que sus aportaciones han influido en la percepción y el análisis en esta materia; tal es el caso de los trabajos realizados por María Ángeles Durán, Teresa Rendón y Mercedes Pedrero, que han sido una importante guía y un referente en la región. También se refirió a las organizaciones de expertas y expertos en encuestas sobre uso del tiempo y trabajo no remunerado, que desde 2010 se realizan anualmente y donde la discusión y la reflexión académica han sido muy importantes. Un caso particular al que se refirió es a la alianza que han establecido ONU Mujeres y El Colegio de México. Explicó que 
el objetivo central de esta alianza ha sido motivar la discusión y la reflexión con base en análisis de calidad para tener mayor incidencia en las políticas públicas. El propósito de esta colaboración ha sido asimismo incentivar el interés sobre el tema en las nuevas generaciones con el apoyo de investigadoras e investigadores consolidados que motiven a los estudiantes y a los recién graduados a abocarse al estudio del uso del tiempo y el trabajo no remunerado. El resultado fue una serie de estudios que integran la publicación Uso del tiempo y trabajo no remunerado en México, que coordinaron Brígida García y Edith Pacheco; en ellos se reflexiona sobre el trabajo remunerado y el no remunerado, sobre la pobreza de tiempo, sobre los vínculos del trabajo no remunerado con la dinámica de los hogares, y sobre el uso del tiempo de acuerdo con el ámbito de residencia y la condición indígena, entre otros temas relevantes.

Indicó que los avances han sido notables, pues actualmente se tiene un mayor conocimiento sobre el valor del trabajo no remunerado y su contribución a la economía global; sobre la pobreza de tiempo de las mujeres; sobre lo que significa para ellas la carga del trabajo no remunerado; sobre las desigualdades en la vida laboral y doméstica entre mujeres y hombres; sobre la discriminación laboral y salarial, y sobre los trabajos que necesitan y demandan las mujeres. Sin embargo, reconoció que aún no se han llevado a la práctica acciones de política efectivas que permitan la conciliación de la vida laboral y familiar con corresponsabilidad social. Al respecto, un tema que adquiere relevancia, dada la transición demográfica, es el de las políticas de cuidado. Finalmente, María de la Paz anotó que: "como decía con frecuencia nuestra directora ejecutiva, la señora Michelle Bachelet, necesitamos nuevos argumentos para políticas públicas sustentados en evidencias empíricas. En esto el trabajo de la academia resulta imprescindible; de esta manera no se trata simplemente de celebrar, sino de detenerse a mirar nuestros logros, pero también de centrar la mirada ahora en los desafíos". 


\section{INFORMES}

\section{Intervención de María Ángeles Durán²}

María Ángeles Durán inició su intervención con algunos comentarios puntuales sobre los elementos a considerar en el diseño de las agendas futuras de investigación sobre trabajo no remunerado y familia. Primero se refirió a la agenda como una coincidencia de propósitos en un cronograma, lo cual implica fijar unas fechas y proponerse ciertos avances para entonces. En segundo término enfatizó que para que esa agenda se lleve a cabo es necesario involucrar y comprometer a tres actores: los investigadores, los patrocinadores económicos y un actor social o político. Algunas veces todos son los mismos, otras los investigadores son actores sociales y políticos, otras más quienes fungen como investigadores sociales en realidad desempeñan una gerencia de investigador o tienen un cargo político y a veces el político actúa como el investigador y se preocupa no sólo por el poder sino también por la sociedad. Aquí mencionó que los investigadores ofrecen su capacidad de trabajo y estimulan el proceso, y a veces incluso pueden inducirlo, mientras que el actor social está preocupado sobre todo por el cambio social. De modo que para conseguir que haya agendas es necesario trabajar distintos frentes simultáneamente. En tercer término se refirió a la importancia de tomar riesgos en las agendas, sobre todo entre la generación de jóvenes investigadores, quienes tienen que saber qué cantidad de riesgo quieren incorporar a los tópicos con los que trabajan: riesgo por los temas, por los destinatarios y por la posibilidad de conseguir financiamiento. Como cuarto punto destacó la importancia de formular una buena estrategia, y con ello se refirió a la formación de redes de trabajo en la medida en que sea mayor la capacidad de dar a conocer el tema. Aquí celebró las alianzas para la investigación que ONU Mujeres y El Colegio de México han establecido y que se reflejan en los resultados de investigación sobre el trabajo no remunerado.

El siguiente elemento que Durán refirió es el tipo de agenda que se está pensando, de carácter local, nacional o regional. En el sexto

\footnotetext{
${ }^{2}$ Pionera y experta en la investigación sobre trabajo no remunerado. Es doctora honoris causa por las universidades autónomas de Madrid, Valencia y Granada. Es presidenta honoraria de la Cátedra UNESCO de "Políticas de igualdad" en la Universidad Autónoma de Madrid (UAM). Ha sido profesora visitante en Michigan, Río de Janeiro, Cambridge, Washington y en el Instituto Europeo de Florencia; además es miembro del Executive Committee de la International Sociological Association. Colabora frecuentemente con universidades y organismos latinoamericanos, entre otros CEPAL, ONU Mujeres y la Organización Panamericana de la Salud (OPS). Su libro más reciente es El trabajo no remunerado en la economía global.
} 
punto argumentó que es preciso identificar si el proyecto de que se trata es de corto, mediano o largo plazos, ya que no es lo mismo formular agendas para abordar problemas que se pueden resolver enseguida, que hacerlo para cuestiones que sólo se podrán resolver en mediano o largo plazos. Al respecto dijo que "las cosas relevantes hay que por lo menos pensarlas a cinco, diez y quince años”. Como séptimo punto recomendó que se tenga en cuenta la transmisión generacional y se reconozca que el ciclo del investigador individual es corto, mientras que el de las escuelas es un ciclo largo. Los grandes avances no los suele lograr una sola persona, sino una que forma parte de un equipo, que hace alianzas y así entre todos forman un movimiento intelectual. Destacó que la investigación sobre trabajo no remunerado es un movimiento social e intelectual, es un movimiento social que le da gran fuerza política y que hizo posible que la ONU en la Cuarta Conferencia Mundial sobre la Mujer en septiembre de 1995 en Beijing cambiara el marco de análisis macroeconómico.

La expositora argumentó que otro elemento que es importante considerar en el diseño de la agenda es que la ciencia es una actividad domeñada y el que quiera innovar va a tener que pelear por abrirle nuevos brazos. Como noveno punto, dijo que es importante que la investigación, además de obtener resultados muy concretos de cifras basadas en la observación extensiva, sea mucho más humanista para que se debata la división del trabajo por sexo, edades, etnias y clases sociales.

También María Ángeles Durán hizo referencia a los temas de la agenda futura de investigación sobre trabajo no remunerado, esencialmente a los retos que plantea el proceso de envejecimiento. Señaló que el tiempo no se puede seguir pensando como si fuera el de las épocas en que la población era joven; debe tomarse en cuenta que hay una proporción demográfica distinta de la población, debe pensarse en un nuevo Estado que provea los servicios que requiere una estructura envejecida. Asimismo resaltó la importancia de la contabilidad nacional, ya que es la herramienta más importante para adoptar decisiones de política pública. Como parte de la agenda futura requiere partir de una perspectiva macroeconómica, con un nuevo tipo de interpretación sobre lo que es producir y lo que es consumir.

Apuntó que algunos países de Latinoamérica como Cuba y Uruguay enfrentan hoy el desafío de un acelerado envejecimiento poblacional, mientras que Argentina y Chile ya están empezando a envejecer. Por su parte, si bien México es actualmente un país de jóvenes, 


\section{INFORMES}

se anticipa que para el año 2050 será una nación envejecida. La cuestión es muy relevante porque en el sistema de producción capitalista de bienes quienes llegan a una mayor edad tienen que salir del mercado, pero como cada vez se prolonga más su esperanza de vida, ¿quién pagará sus pensiones?, ¿deberán retirarse forzosamente de la actividad laboral aun cuando puedan y quieran seguir trabajando?, ¿se estará considerando que vivirán más horas vacías, no libres sino vacías?, ¿el adulto mayor será sólo un consumidor, o tendrá que desarrollar una capacidad de producir para él y quizá para otros?, ¿Quién cuidará de esas personas?

La experta en investigación sobre trabajo no remunerado advirtió que si se está pensando que las mujeres sean las cuidadoras de la población adulta mayor, se deberá tener en cuenta que ellas querrán aumentar su renta per cápita y estar en el mercado de trabajo remunerado, lo cual les conviene porque las hace más autónomas y les da derechos para la vejez. Si se piensa que las mujeres que se quedaron en casa se harán cargo de los cuidados, entonces habrá que tener en cuenta el aumento en la carga de trabajo no remunerado. ¿Será la población adulta mayor la que cuidará sin cobrar?, ¿habrá servicios públicos gratuitos?, ¿vamos a depender del mercado?, ¿pueden pagar sus cuidados el niño, el que lleva mucho tiempo enfermo, o el que está muy frágil porque es anciano? María Ángeles Durán finalizó su intervención advirtiendo que "el cuidado es un tema político de primera magnitud y además es un tema humano que merece que los investigadores colaboremos en anticipar soluciones antes de que tengamos el problema doliéndonos a todas horas, en nuestra casa y en las personas que queremos".

\section{Intervención de Paulo Saad ${ }^{3}$}

La intervención de Paulo Saad tuvo por título "Bono de género y redistribución del trabajo no remunerado". Ahí expuso que su investigación se ha desarrollado en un contexto donde aún persisten desigualdades importantes entre mujeres y hombres y donde se aproxima

${ }^{3}$ Es jefe del Área de Población y Desarrollo de Celade, División de Población de la CEPAL de Naciones Unidas. Es doctor en Sociología por la Universidad de Texas en Austin, maestro en Demografía por El Colegio de México y bachiller en Estadística por la Universidad de São Paulo en Brasil. Trabajó por ocho años en la División de Población del Departamento de Asuntos Económicos y Sociales de Naciones Unidas en Nueva York, antes de ser transferido a la CEPAL en octubre de 2007. 
el fin del periodo del bono demográfico en los países de América Latina. En relación con las desigualdades de género se refirió a la rigidez de la distribución sexual del trabajo, que genera una inserción desventajosa de las mujeres en el mercado laboral; al uso diferencial del tiempo; a la sobrecarga femenina en la provisión de cuidado, sea como cuidadoras de niños, personas mayores o enfermos. Indicó que son pocos los países de la región que tienen una agenda clara en la política de protección social, es decir, donde la familia, la comunidad, el Estado y el mercado participen de manera equilibrada. Enfatizó que las soluciones que se plantean desde el mercado sólo benefician a un grupo específico de la población, lo que genera desigualdad. Advirtió que las políticas de cuidado ocupan un lugar secundario; que se diseñan como si se tratara de un beneficio para las mujeres ocasionando así que su trabajo se devalúe, y generando una limitación para que el cuidado se asuma como una responsabilidad compartida. Además llamó la atención sobre las medidas laborales que generan la precarización laboral y fortalecen la responsabilidad de las mujeres en las tareas de cuidado.

Respecto al fin del periodo del bono demográfico mencionó que en países como Cuba y Chile ha terminado recientemente el periodo en que la relación de dependencia se ha mantenido decreciente, si bien en Bolivia y Guatemala perdurará por unas cuatro décadas más, en general el bono demográfico habrá terminado en las próximas tres décadas en prácticamente todos los países de la región. Hizo referencia al Índice de Dependencia Económica (IDE) como una manera alternativa de estimar el efecto económico de los cambios en la estructura etaria; es un indicador que toma en cuenta los factores demográficos y además la inserción de las personas en el mercado de trabajo. En todos los países de América Latina el IDE tiene un periodo favorable que se extiende más allá del correspondiente al bono demográfico. Paulo Saad se refirió a cuatro fechas emblemáticas que plantean desafíos económicos importantes para el caso de México: 1) En 2018 la razón de soporte fiscal (contribuyentes / beneficiarios) comenzará a descender de manera ininterrumpida. 2) Se proyecta que en 2027 los jóvenes dejarán de ser el grupo demográfico dominante, lo cual marcará el fin de la sociedad joven en México. 3) En 2033 la razón de soporte económico (productores / consumidores) alcanzará el punto máximo y comenzará a disminuir. 4) En 2035 la economía del país se volverá envejecida y por primera vez el consumo de las personas mayores superará al de los jóvenes. 


\section{INFORMES}

Saad, jefe del Área de Población y Desarrollo de Celade, definió el bono de género como "el aumento de la producción económica per cápita que se genera conforme se avanza hacia la paridad entre mujeres y hombres en el mercado de trabajo, lo que supone la superación de barreras discriminatorias en contra de ellas, existentes tanto dentro como fuera de este mercado". Mencionó además las ventajas derivadas del análisis y la evaluación del bono de género: $a$ ) es una fuente de recursos económicos que los países pueden potenciar mediante políticas adecuadas; $b$ ) da cuenta de la importancia de la equidad de género en el logro del desarrollo sostenible; $c$ ) brinda soporte a la necesidad de incluir un enfoque de género en las políticas públicas dirigidas a igualar las oportunidades de las mujeres en el mercado de trabajo; y d) añade elementos relevantes en el análisis de las relaciones entre la dinámica poblacional y el desarrollo.

El expositor explicó detalladamente la metodología que debe seguirse para la medición de la magnitud del bono de género. En principio, el crecimiento del PIB per cápita se calcula como la suma de cuatro factores: $s=$ tasa de crecimiento del producto por trabajador, $t=$ tasa de crecimiento de la participación laboral en las edades laborales, $u=$ tasa de crecimiento de la población en las edades de trabajar y $n=$ tasa de crecimiento de la población total. El bono demográfico se mide como la diferencia entre la tasa de crecimiento de la población en edad de trabajar y la población total $(u-n)$, mientras que el bono de género se mide por la tasa de crecimiento de la tasa de participación laboral en las edades de trabajar. Aquí aclaró que esta aproximación se realiza porque la mayor parte de la tasa de crecimiento se debe a cambios en la tasa de actividad femenina. Además dijo que se trata de un bono de género potencial, ya que la medida exagera la contribución económica real de las mujeres, puesto que aún persisten brechas salariales importantes entre mujeres y hombres.

Entre los resultados de la investigación Saad señaló que el impacto del bono de género sobre el crecimiento del PIB per cápita es considerable. En el periodo de 1980-2010 para todos los países de América Latina la contribución al crecimiento anual del PIB per cápita tanto del bono de género como del bono demográfico fue de aproximadamente 0.6 puntos porcentuales. Sin embargo, refirió diferencias importantes entre los países: en Bolivia, Colombia, Guatemala, Argentina y Ecuador los bonos de género contribuyeron en más de 0.8 puntos porcentuales, mientras que en Cuba y Haití dichas contribuciones fueron inferiores a 0.2 puntos porcentuales y en México fueron supe- 
riores a 0.8 puntos porcentuales. $\mathrm{Al}$ analizar la contribución anual de ambos bonos en el periodo 2010-2040 dijo que se espera una disminución del tamaño del bono demográfico en la mayoría de los países de la región (0.3 puntos porcentuales), en tanto que la contribución del bono de género también se reducirá (0.4 puntos porcentuales), pero en promedio se proyecta que el bono de género excederá al bono demográfico en 12 de los 20 países de la región. México es uno de los países en donde el dividendo de género será más importante.

A manera de conclusión, Saad mencionó que si bien ha sido mayor la participación económica de las mujeres en América Latina, aún persisten brechas de género en las actividades económicas, incluso en los países de la región con mayores niveles de equidad. Resaltó que todavía hay un amplio margen para el aprovechamiento del bono de género, que su magnitud y duración dependen de una serie de factores que se relacionan con la distribución de los roles en el interior de los hogares y con el funcionamiento del mercado de trabajo. Hizo un llamado para emprender acciones encaminadas a redistribuir tanto el trabajo remunerado como el no remunerado, especialmente aquellas actividades de cuidado que socialmente han sido asignadas a las mujeres. Además enfatizó la necesidad de que el Estado, el mercado y la comunidad participen en esta redistribución del trabajo. Habló de la relevancia de aumentar la inserción laboral de la población femenina y disolver la segmentación ocupacional de las mujeres.

Para concluir su participación Saad afirmó que: "quienes diseñan y analizan las políticas sociales aún no advierten que la responsabilidad compartida de cuidado, y la igual participación de mujeres y hombres en todas las esferas dará frutos mejores y más duraderos de desarrollo humano y económico".

\section{Comentarios}

Después de las tres exposiciones, Edith Pacheco celebró la próxima publicación del libro Uso del tiempo y trabajo no remunerado en México, agradeció la presencia de las autoras y los autores y refirió que la obra presenta un estudio cuidadoso y detallado del uso del tiempo en México durante los últimos años del siglo XXI. Se compone de 11 capítulos de amplio alcance que abordan una temática de frontera y son pioneros en el estudio sobre uso de tiempo en México y en América Latina: 


\section{INFORMES}

Capítulo I. A manera de introducción, las coordinadoras Brígida García y Edith Pacheco dan cuenta de las principales contribuciones de las y los autores y comparten una detallada reflexión conceptual y metodológica sobre el estudio del tiempo.

Capítulo II. Mercedes Pedrero elabora una minuciosa discusión conceptual sobre la importancia del trabajo no remunerado, así como su medición y valoración a través de las encuestas de uso del tiempo.

Capítulo III. Araceli Damián aborda la discusión sobre la captación del uso de tiempo y la medición de la pobreza de tiempo.

Capitulo IV. Laura Santoyo y Edith Pacheco analizan el uso del tiempo a partir de la organización de los hogares.

Capítulo V. Estela Rivero y Anairis Hernández revisan los diferentes patrones de organización del tiempo y exploran si existe o no un intercambio entre las distintas tareas.

Capitulo VI. Nelson Florez y Edith Pacheco hacen una revisión de las desigualdades de tiempo entre las mujeres y los hombres en dos contextos con características sociales, culturales y económicas completamente diferenciadas: lo rural y lo urbano.

Capítulo VII. Teresa Jácome y Marta Mier y Terán examinan el uso del tiempo entre la población indígena.

Capítulo VIII. Mauricio Rodríguez y Brígida García analizan las actividades domésticas y de cuidado que los varones mexicanos desempeñan, así como los factores que determinan la cantidad de tiempo dedicado a estas tareas.

Capítulo IX. Olga Rojas y Mario Martínez se refieren al uso del tiempo entre los padres mexicanos: el que destinan al trabajo doméstico y a la crianza y cuidado de los hijos.

Capítulo X. Landy Sánchez examina la brecha de género en el trabajo doméstico en las parejas de doble ingreso.

Capítulo XI. Clara Márquez y Minor Mora analizan las inequidades de género y los patrones de uso de tiempo entre la población que manifiesta estar disponible para integrarse al mercado laboral.

\section{Elementos para la agenda futura de investigación}

Tras las presentaciones se organizó una nutrida discusión sobre los próximos pasos en las investigaciones y sobre los elementos que deben integrarse en la agenda futura de estudios sobre trabajo no remunerado y familia. Uno de los temas más debatidos fue el bono de género; 
se habló sobre la desigualdad socioeconómica y la heterogeneidad dentro de los mercados de trabajo, elementos que anulan los efectos del bono. Sin embargo, se enfatizó que el concepto de bono se refiere a una situación coyuntural potencial que depende en gran medida de las políticas que se implementen para aprovechar esa situación.

Entre los participantes hubo consenso acerca de que las políticas públicas y los programas institucionales no siempre son acertados y suficientes e incluso muchas veces contribuyen a perpetuar los roles de cuidadoras entre las mujeres. Por otro lado, es común que ellas asuman dobles cargas de trabajo. Es preciso estar atentos a las trampas invisibles, ya que no siempre la curva de bienestar mejora cuando aumenta el trabajo remunerado; no siempre equidad significa igualdad. Además, se reconoció la importancia de tomar en cuenta las características económicas, sociales y culturales específicas de cada país, así como el impacto real de las políticas sobre la calidad de vida de la mujer. Se dijo que lo recomendable sería que todas las políticas se analizaran en términos de costos de tiempo y se incluyeran en ellas los distintos grupos vulnerables de la población, como las personas en edades avanzadas. También se mencionó la importancia de no reducir el análisis del bono de género a un enfoque productivista que sólo tome en cuenta el trabajo que tiene un valor económico, sino que el enfoque de análisis debe ser integral para considerar los aportes económicos tanto del trabajo remunerado como del trabajo doméstico y de cuidados que no está remunerado.

Hubo consenso en la necesidad de homologar conceptos y de realizar análisis más profundos en las agendas futuras. Por ejemplo, se sugirió que en el estudio del tiempo se analicen con detalle sus dimensiones teórica, conceptual y filosófica. Un tema que es necesario resignificar es el ocio, pues los datos existentes aún son incompletos, no queda claro si se habla de tiempo libre o de ocio, y dentro del ocio si es activo o pasivo, y sobre este último si es tiempo de exclusión o tiempo de cansancio y enfermedad. Asimismo se enfatizó sobre la importancia de que la investigación sobre trabajo no remunerado se realice en el marco de un mundo globalizado, ya que hay que considerar siempre que las cadenas globales de cuidado son de carácter transnacional. Por otro lado, en la propuesta de las iniciativas de cambio social la agenda debe incluir un pacto de género y de generaciones. Todos se podrían beneficiar más si participaran en ambos mercados.

Se propuso que en un lapso no mayor de cinco años toda América Latina cuente con cuentas satélites del trabajo no remunerado. La 


\section{INFORMES}

evolución de las encuestas de uso del tiempo en América Latina denota que esto sí se puede concretar, si bien no íntegramente, pero constituirá un avance de amplio impacto social y político porque proporcionará los instrumentos que resultan clave en la negociación. También se sugirió crear una red de investigadores con miras a encontrar múltiples puntos de consenso.

Finalmente se destacó que el "poder de las ideas" que surge de los trabajos de investigación es un mecanismo fundamental para persuadir a la sociedad de la importancia de la contribución del trabajo no remunerado. 
